ISSN: 2637-7764

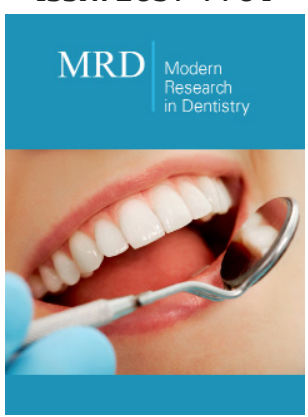

*Corresponding author: Márcia Rodrigues P, School of Dentristy, Oral Pathology Division, Brazil

Submission: 䟧April 30, 2019

Published: : May 08, 2019

Volume 4 - Issue 1

How to cite this article: PAYERAS M R, LOPES T G, CHERUBINI K, FIGUEIREDO M A Z, SALUM F G. Angiogenesis in Oral Lichen Planus: A Comparative Study with Lesions of Different Degrees of Aggressiveness. Mod Res Dent. 4(1). MRD.000578.2019. DOI: 10.31031/MRD.2019.04.000578

Copyright@ Márcia Rodrigues P, This article is distributed under the terms of the Creative Commons Attribution 4.0 International License, which permits unrestricted use and redistribution provided that the original author and source are credited.

\section{Angiogenesis in Oral Lichen Planus: A Comparative Study with Lesions of Different Degrees of Aggressiveness}

\author{
Márcia R Payeras ${ }^{1 *}$, Tiago G Lopes ${ }^{2}$, Vinícius D Silva ${ }^{3}$, Karen Cherubini ${ }^{4}$, Anto- \\ nia Zancanaro FM ${ }^{4}$ and Fernanda G Salum ${ }^{4}$ \\ ${ }^{1}$ School of Dentristy, Oral Pathology Division, Brazil \\ ${ }^{2}$ PPGCM, Brazil \\ ${ }^{3}$ Cancer Hospital of Barretos, Brazil \\ ${ }^{4}$ School of Health Sciences, Oral Medicine Division, Brazil
}

\begin{abstract}
Background: The immunodetection of VEGF, ANG-1 and 2 was investigated in oral lichen planus (OLP) and in control groups to compare angiogenesis between lesions with different degrees of aggressiveness.

Methods: A cross-sectional observational study was performed in 21 reticular (ROLP) and 11 atrophicerosive (AEOLP) specimens of OLP and in 10 cases of oral fibroepithelial hyperplasia (OFH), oral epithelial dysplasia (OED) and oral squamous cell carcinoma (OSCC).

Results: The results do not revealed significant difference in the immunodetection of ANG-1 and ANG-2 between OLP groups. The ROLP group showed significantly greater immunodetection of VEGF compared with the EOLP group. The comparison between the OLP group and each control group showed significantly greater levels of ANG-1 in the OLP group compared to the OFH group and no significant difference in ANG-2. VEGF levels in the OLP group were significantly higher than in the OFH group and significantly lower compared to the OSCC group.
\end{abstract}

Conclusion: In this small sample, the results indicate that angiogenesis in OLP cannot be associated to the different clinical forms of this disease. The lack of correlation between the markers in the OLP groups suggests the involvement of other pro-angiogenic agents and reflect the complexity this process and the necessity for further studies. Immunodetection of these growth factors in the OLP and OED groups was similar, demonstrating that angiogenesis in OLP may behave similarly to OED.

Keywords: Oral lichen planus; Angiogenesis; Vegf; Ang-1; Ang-2

\section{Introduction}

Angiogenesis, the process by which new vessels are formed from pre-existing vascular structures, is controlled by numerous growth factors and pro-angiogenic cytokines, as well as by various endogenous inhibitors of neovascularization. under physiological situations, these activators and inhibitors are in balance, but in pathological situations there can be persistent or excessive activation of pro-angiogenic agents [1]. Vascular endothelial growth factor (VEGF) is one of the most potent and known stimulators of angiogenesis, showing the ability to induce vascular permeability [2], besides the proliferation and migration of endothelial cells, essential steps for the development of new blood vessels [3]. Other growth factors essential for vascular formation are the angiopoietins (ANG), among which ANG-1 and ANG-2 are the most known and best characterized [4]. In contrast to VEGF, angiopoietins are angiogenic mediators whose action does not induce mitotic activity in endothelial cells [5]. Despite their biological activities not being completely elucidated, it is known that ANG-1 is constantly secreted in situations of vascular quiescence, as well as during the process of vascular maturation, a later step in angiogenesis [5,6]. Besides, ANG-1 acts as an anti-inflammatory cytokine [4]. On the other hand, ANG-2, despite showing affinity for the same Tie-2 receptor of ANG-1, does not phosphorylate it, thus exerting an antagonistic effect of ANG-1 [4], destabilizing the vascular endothelium, which causes the regression of neoformed vessels and apoptosis in endothelial 
cells [7]. However, some studies have identified ANG-2 as an agonist for the Tie- 2 receptor, inducing angiogenesis and the inflammatory response [6], when this receptor is expressed in non-endothelial cells [7] or in the presence of VEGF [8].

In chronic inflammatory diseases such as rheumatoid arthritis [5] investigations have revealed a strict relation between angiogenesis and disease activity, which contributes to its persistence and chronicity. In addition, it has been demonstrated that angiogenesis it related to cell proliferation [9] and can be an early event in malignant transformation [10]. Oral lichen planus (OLP) is a chronic inflammatory disease of unknown etiology and uncertain pathogenesis and although its risk of malignant transformation is low [11], it is considered a potentially malignant disease [12]. Few studies have investigated angiogenesis in OLP [13-16], but it is believed that the rich vascularization present in the lesions probably occurs as a result of hypoxia, caused by the proliferation of lymphocytes [17]. Recent studies have demonstrated an increase in the expression of VEGF [16] and in micro vessel density (MVD) [13-15] in OLP, especially in the erosive form, suggesting that angiogenesis can play a distinct role in the different clinical forms of this disease [13]. In this study, the immunodetection of VEGF, ANG-1 and ANG-2, as well as the number of blood vessels was investigated in specimens of reticular and atrophic-erosive OLP. Besides, these markers were determined in specimens of oral fibroepithelial hyperplasia, oral epithelial dysplasia and oral squamous cell carcinoma and compared with values present in OLP, in order to find a relation between those angiogenic growth factors and biological behavior.

\section{Materials and Methods}

This study was approved by the Committee of Ethics in Research of the Pontifical Catholic University of Rio Grande do Sul (PUCRS), under protocol 11/05540.

\section{Tissue samples}

Specimens of biopsies embedded in paraffin were obtained from adult patients of both sexes. According to the clinical and histopathological diagnosis, based on WHO [18] criteria, the sample was distributed into the following groups: OLP, 32 specimens of oral lichen planus, subdivided into ROLP with 21 specimens of reticular oral lichen planus and AEOLP with 11 specimens of atrophic or erosive oral lichen planus; OFH, 10 specimens of oral fibroepithelial hyperplasia; OED, 10 specimens of oral epithelial dysplasia; and OSCC, 10 specimens of oral squamous cell carcinoma. The inclusion criteria utilized for the selection of cases of ROLP were the presence of reticular, plaque or papule patterns and, on microscopic examination, inflammatory infiltrate composed mainly of lymphocytes, arranged in a band in the subepithelial region, signs of hydropic degeneration in the basal layer and absence of epithelial dysplasia. In the diagnosis of AEOLP, the clinical requirement was the presence of erosive or atrophic types and histopathological evidence of atrophic or ulcerated epithelium, besides the above characteristics. When there was an association between white and atrophic/erosive lesions, they were inserted in the AEOLP group, since these areas were included in biopsies. The lesions of the OFH group were required to microscopically show fibroplasia, hyperplasia of epithelial lining, and also lymphoplasmacytic infiltrate and vascularization in the lamina propria. The cases of OED were included when there was clinical manifestation of leukoplakia, erythroplakia or erythroleukoplakia and microscopic evidence of epithelial dysplasia. Inclusion in the OSCC group required epithelial neoplastic lesions to appear moderately differentiated. Biopsy specimens in which it was not possible to perform all steps in the immunohistochemical assay were excluded. The medical charts were analyzed and data such as gender, age and localization were recorded, even as clinical pattern and number of sites affected, in the cases of OLP.

\section{Immunohistochemistry}

Three new sections were obtained from each sample, with a thickness of $3 \mu \mathrm{m}$, which were deparaffinized in xylene and rehydrated in alcohol. Endogenous peroxidase was blocked by immersing the sections in $0.3 \%$ hydrogen peroxide in methanol. Antigen retrieval was done with citrate buffer, pH 6.0 (Dako Corporation, Carpinteria, CA, USA) in a steamer for 20 minutes. Next, the sections were cooled to ambient temperature. The slides were then incubated in an oven at $30^{\circ} \mathrm{C}$ for 1 hour with the primary antibodies anti-VEGF (1:200; monoclonal antibody, anti-human, Santa Cruz Biotechnology, CA, USA), anti-ANG-1 (1:100; polyclonal antibody, anti-human, R\&D System, Minneapolis, MN, USA) and anti-ANG-2 (1:50; polyclonal antibody, anti-human, R\&D System, Minneapolis, MN, USA). Primary antibodies were revealed using the avidin-biotin system (Dako Corporation, Carpinteria, CA, USA) with 45 minutes incubation at $30^{\circ} \mathrm{C}$. The sections were counterstained with Harris' hematoxylin and mounted with Entellan (Merck KgaA, Darmstadt, Germany). Amygdala was used as a positive control for VEGF and prostate was used for ANG-1 and ANG-2. The primary antibody was omitted in the negative control.

\section{Immunodetection of VEGF, ANG-1 and ANG-2}

The immunodetection of the growth factors evaluated was visualized by the presence of a brown color in the tissues. When immunodetection was positive, the structures evaluated were epithelial tissue, connective tissue with its cellular elements (fibroblasts and inflammatory cells) and blood vessels. The quantitative determination of the antigens VEGF, ANG-1 and ANG-2 was carried out by a single blinded observer, in ten microscopic fields captured at equidistant points on each slide at 200x magnification. A Zeiss Axioskop 40 light microscope (Zeiss; Oberkochen, Germany) was used coupled to a Roper Scientific videocamera (Media Cybernetics, Silver Springs, MD, USA) and a Pentium IV $2.2 \mathrm{GHz}$ computer with $512 \mathrm{MB}$. The images captured were later transferred to the software Image J for Mac, version 1.47a (CyberMedia, NY, USA). In each image, the percentage of area showing positive staining was calculated by the semi-automated segmentation technique. The number of blood vessels was determined in slides immunostained for ANG-1, utilizing the same software, by means of a manual counting tool. Structures in which the presence of endothelium was evident were considered vessels. 


\section{Statistical analysis}

The data were processed and analyzed using the statistical software SPSS for Windows, version 17.0. P-values $\leq 0.05$ were considered significant. The data were initially analyzed by means of descriptive statistics. Student's t-test for independent samples was applied for comparison of immunodetection of VEGF between the NEOLP and EOLP groups. This test was also utilized to compare the immunodetection of VEGF between the OLP group and each of the control groups. Comparison of expression of ANG-1 and ANG2 between the ROLP and AEOLP groups was carried out using the non-parametric Mann-Whitney test, which was also employed for comparing the OLP group with each of the control groups. The comparison of the number of vessels between the ROLP and AEOLP groups was done using the non-parametric Mann-Whitney test. The correlation between the expression levels of VEGF, ANG-1 and ANG2 in different lesions studied was evaluated utilizing the Spearman Correlation coefficient.

\section{Result}

\section{Sample characterization}

The mean age of the ROLP group was $51.44 \pm 11.91$ years and of the AEOLP group $50.60 \pm 10.51$ years. Females predominated in the ROLP and AEOLP groups, corresponding to $14(66.66 \%)$ and $8(72.72 \%)$ cases, respectively. In the ROLP group, 9 (42.85\%) cases showed the presence of more than one clinical pattern, with a predominance of the reticular pattern, alone or combined in 15 (71.42\%). With respect to the AEOLP group, 9 (81.81\%) patients also displayed more than one clinical pattern. The reticular pattern was present associated with atrophic-erosive lesions in 7 (63.63\%) cases. In regard to localization, both OLP groups exhibited a predominance of lesions at more than one site and the most affected was the buccal mucosa, which corresponded to 11 (52.38\%) cases in the ROLP group and 9 (81.81\%) cases in the AEOLP group. In relation to the control groups, it was noted that the OFH group showed a mean age of $58 \pm 11.91$ years, the OED group $53.44 \pm 16.23$ years, and the OSCC group $56.8 \pm 15.23$ years. Among these, the OFH and OED groups showed a predominance of female patients, with $6(60 \%)$ and $8(80 \%)$ cases, respectively. In the OSCC group, there was a predominance of males, corresponding to 8 (80\%) cases.

\section{Vegf, ang-1 and ang-2 immunodetection}

Immunodetection of $\mathrm{VEGF}$ and $\mathrm{ANG}-2$ was observed in epithelial, endothelial and connective tissue cells. On the other hand, ANG-1 was detected mainly in epithelial and endothelial cells, while there was discrete staining of fibroblasts (Figure 1-3). With regard to the type of staining present in the epithelium, immunodetection of ANG-1 was observed mainly in the membrane and cytoplasm (Figure 1). ANG-2 and VEGF immunostaining was predominantly localized in the cytoplasm and nucleus (Figure 2 \& 3 ) in all the groups studied. In connective tissue, immunodetection of VEGF and ANG-2 was observed in fibroblasts and inflammatory cells in all cases. In relation to the percentages of immunodetection, values of ANG-1 were lower compared to the VEGF and ANG-2 in all the groups. Immunodetection of VEGF, ANG-1 and ANG-2 in the ROLP and AEOLP groups is described in Table 1. The ROLP group showed a significantly higher percentage of immunodetection for VEGF $(\mathrm{P}=0.01)$ compared to the AEOLP group, while there was no significant difference between the two groups with respect to ANG-1 and ANG-2. In relation to the number of blood vessels, the comparison between the ROLP and AEOLP groups did not demonstrate a significant difference $(\mathrm{P}=0.393)$. For the comparative analysis between oral lichen planus and control groups, 32 cases of this lesion were considered, which formed the OLP group. When the immunodetection of VEGF was compared between OLP group and control groups, the OLP group showed a significantly higher percentage than the OFH group $(\mathrm{P}=0.003)$ and significantly lower value in relation to the OSCC group ( $\mathrm{P}=0.032)$ (Table 2$)$.

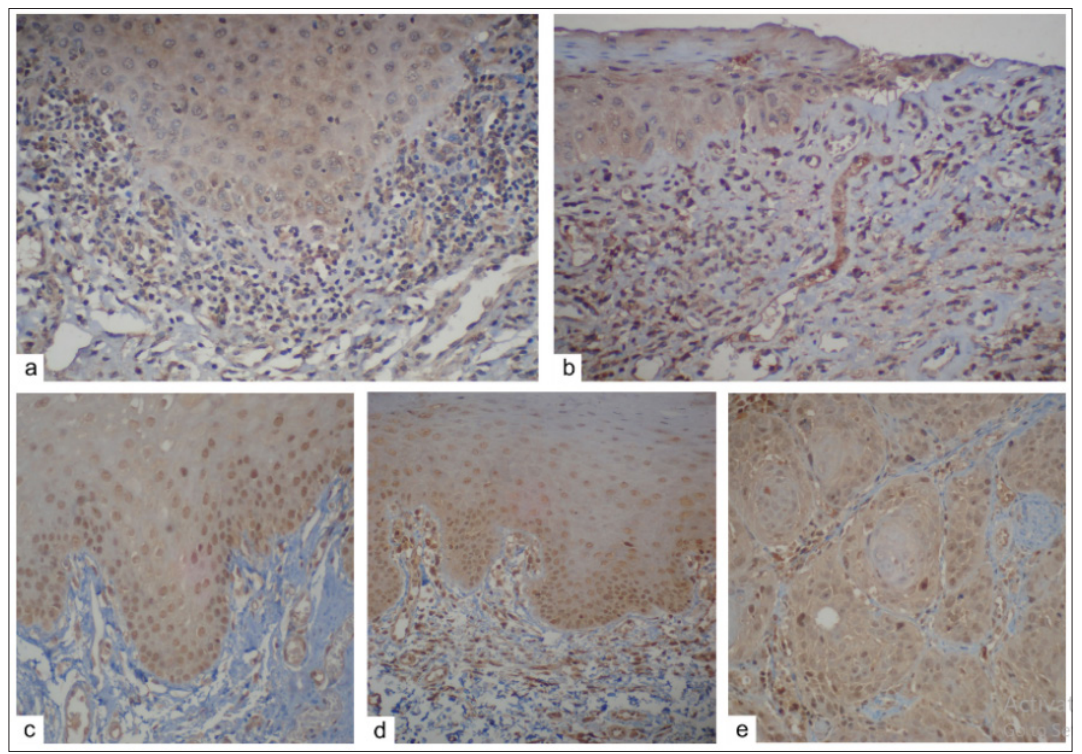

Figure 1: Immunodetection of ANG-1 in specimens of (a): Reticular oral lichen planus (ROLP); (b): Atrophic-Erosive oral lichen planus (AEOLP); (c): Oral fibroepithelial hyperplasia (OFH); (d): Oral epithelial dysplasia (OED); (e): Oral squamous cell carcinoma (OSCC). (Original magnification, x200). 


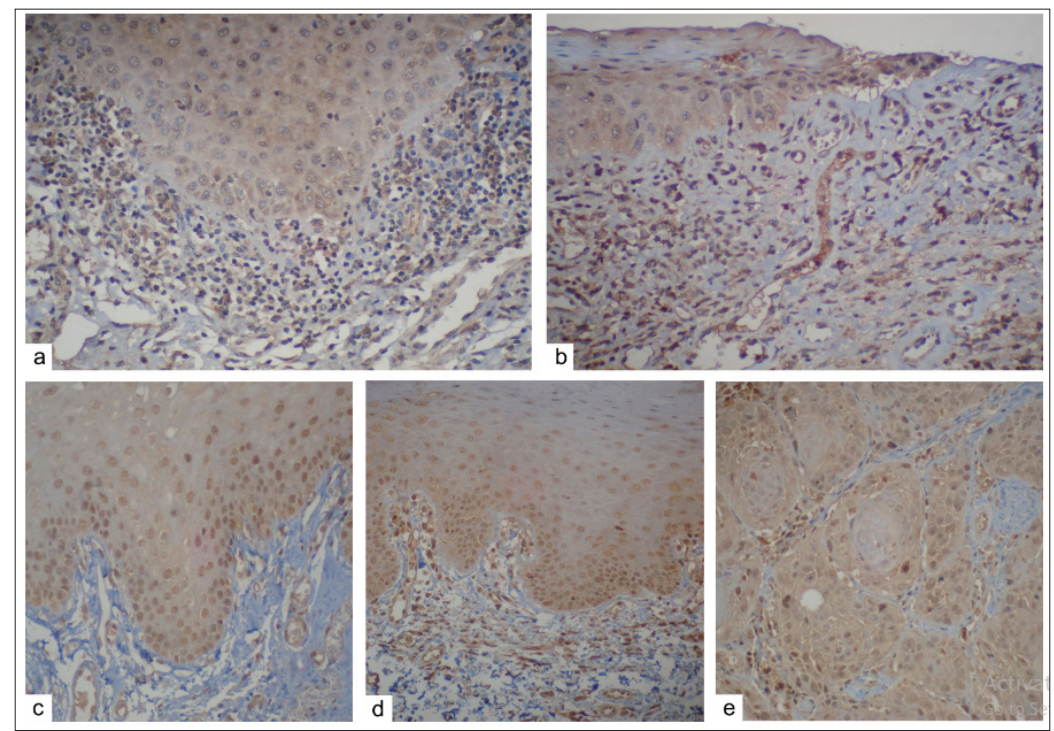

Figure 2: Immunodetection of ANG-2 in specimens of (a): Reticular oral lichen planus (ROLP); (b): Atrophic-Erosive oral lichen planus (EOLP); (c): Oral fibroepithelial hyperplasia (OFH); (d): Oral epithelial dysplasia (OED); (e): Oral squamous cell carcinoma (OSCC). (Original magnification, x200).

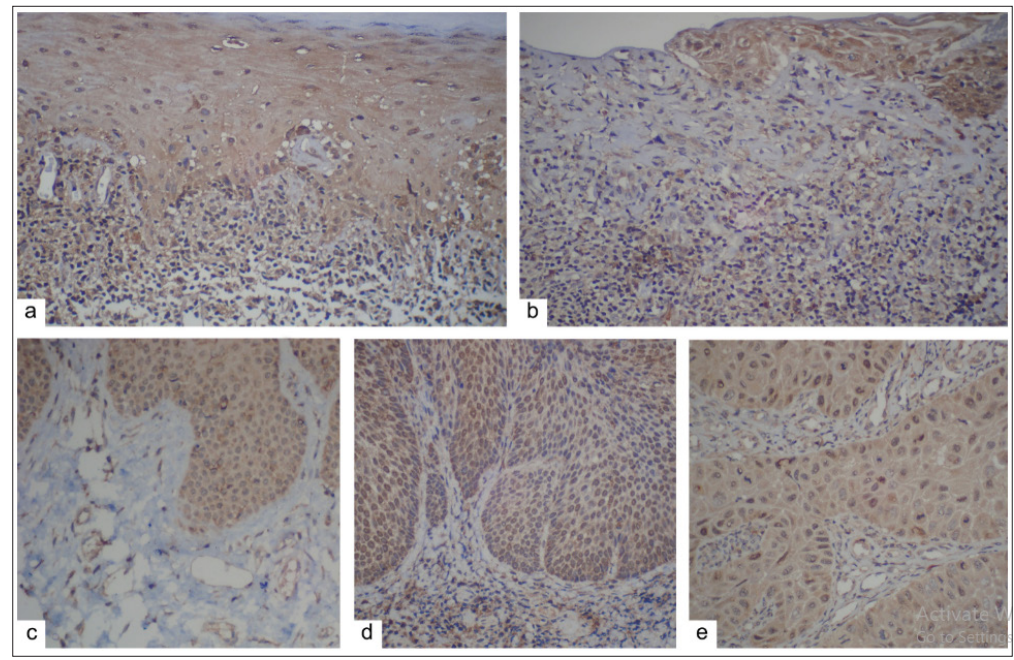

Figure 3: Immunodetection of VEGF in specimens of (a): Reticular oral lichen planus (NEOLP); (b): Atrophic-Erosive oral lichen planus (AEOLP); (c): Oral fibroepithelial hyperplasia (OFH); (d): Oral epithelial dysplasia (OED); (e): Oral squamous cell carcinoma (OSCC). (Original magnification, x200).

Table 1: Mean and standard deviation of the percentage of immunodetection of VEGF and median and interquartile range of the percentage of immunodetection of ANG-1 e ANG-2 in reticular oral lichen planus (NEOLP) and atrophicerosive oral lichen planus (EOLP) groups.

\begin{tabular}{|c|c|c|c|}
\hline \multicolumn{3}{|c|}{ Groups } \\
\hline & ROLP & AEOLP & P \\
\hline VEGF & $46.15 \pm 16.67$ & $28.95 \pm 17.46$ & $0.010^{\mathrm{a}}$ \\
\hline ANG-1 & $11.61(6.61-24.05)$ & $4.55(3.86-23.61)$ & $0.148^{\mathrm{b}}$ \\
\hline ANG-2 & $21.97(10.61-38.19)$ & $27.05(12.46-57.61)$ & $0.372^{\mathrm{b}}$ \\
\hline
\end{tabular}

aStudent T-Test for significance, P-value $\leq 0.05$.

${ }^{\mathrm{b}}$ Mann-Whitney Test for significance, $\mathrm{P}$-value $\leq 0.05$. 
Table 2: Mean and standard deviation of percentage of immunodetection of VEGF in oral lichen planus (OLP) group and control groups.

\begin{tabular}{|c|c|c|c|}
\hline \multirow{2}{*}{ OLP (Mean \pm SD) } & Control groups & Mean \pm SD & P \\
\hline \multirow{3}{*}{$40.23 \pm 18.61$} & Hyperplasia (OFH) & $19.29 \pm 18.56$ & $0.003^{*}$ \\
\cline { 2 - 4 } & & & $48.44 \pm 18.80$ \\
& Dysplasia (OED) & $56.63 \pm 25.52$ & 0.232 \\
\cline { 2 - 4 } & Carcinoma (OSCC) & $0.032^{*}$ \\
\hline
\end{tabular}

*Student T-Test for significance, P-value $\leq 0.05$.

In comparing the immunodetection of ANG-1 between the OLP group and control groups, significantly higher percentages were identified in the OLP group in relation to the OFH group ( $\mathrm{P}=0.043)$. On the other hand, no significant difference was observed in the immunodetection of this marker between OLP group and the control groups OED and OSCC (Table 3). Immunodetection of ANG-2 did not demonstrate a significant difference between the OLP group and the different control groups. However, higher percentages were found in the OFH group, lower levels in the OSCC group, and very similar values between the OLP and OED groups (Table 3). The results did not show any correlation between the immunodetection of VEGF, ANG-1 and ANG-2 in the different groups studied (data not shown).

Table 3: Median and interquartile range of the percentage of immunodetection of ANG-1 e ANG-2 in oral lichen planus (OLP) group and control groups.

\begin{tabular}{|c|c|c|c|c|c|}
\hline & & Median (p25-p75) & Control groups & Median (p25-p75) & $\mathbf{P}$ \\
\hline \multirow{3}{*}{ ANG-1 } & \multirow{3}{*}{ OLP } & \multirow{3}{*}{$10.9(4,54-23,85)$} & Hyperplasia (OFH) & $3.59(1.53-14.98)$ & $0.043^{*}$ \\
\hline & & & Dysplasia (OED) & 7.63 (5.46-18.13) & 0.575 \\
\hline & & & Carcinoma (OSCC) & $5.56(3.76-9.14)$ & 0.104 \\
\hline & \multirow{3}{*}{ OLP } & \multirow{3}{*}{$25.62(11,34-38,90)$} & Hyperplasia (OFH) & 33.55 (17.13-48.17) & 0.256 \\
\hline & & & Dysplasia (OED) & $25.50(11.26-29.40)$ & 0.756 \\
\hline ANG-2 & & & Carcinoma (OSCC) & $16.88(11.92-32.92)$ & 0.535 \\
\hline
\end{tabular}

*Mann-Whitney Test for significance, P-value $\leq 0.05$.

\section{Discussion}

Few studies have investigated angiogenesis in lesions of OLP, and these are limited to evaluations of VEGF, MVD and the adhesion molecules VCAM-1 (Vacular Cell Adesion Molecule-1) and ICAM-1 (Intercellular Adhesion Molecule-1) [13-16]. In the present study, angiogenesis was investigated in OLP by the immunodetection of angiogenic agents that act in different steps in the process of vascular neoformation. VEGF induces an increase in vascular permeability [2] and endothelial proliferation, and it is essential in the initial steps of vascular formation [3]. ANG-1 performs functions related to vascular quiescence, but also to the process of vascular maturation, a later step in the process of the formation of new blood vessels [6]. ANG-2, in turn, destabilizes blood vessels and, depending on the presence or absence of VEGF, induces vascular regression or favors endothelial cell migration, a step also essential for the initiation of angiogenesis [6]. In addition, this study included as control groups oral fibroepithelial hyperplasia, oral epithelial dysplasia and oral squamous cell carcinoma to establish a comparison of angiogenesis in OLP in relation to these lesions, which show different degrees of aggressiveness. We decided to subdivide the OLP group into the reticular and atrophic-erosive forms, since previous studies showed differences in vascularization [13-16], as well as in proliferative activity [19], between these clinical forms, which could be a reflection of the more aggressive behavior of the erosive pattern of the disease, suggested by some authors [20]. However, since there were no significant differences between the ROLP and AEOLP groups in the immunodetection of ANG-1 and ANG-2 and on the basis of the results obtained in relation to VEGF, we decided to form a single OLP group to perform comparative analyses between this disease and control groups. The characteristics of the sample in the different OLP groups were similar to those of previous studies [11,21], where there was predominance of female patients of about 50 years old, who developed mainly reticular lesions localized in the buccal mucosa. These characteristics allowed us to demonstrate that the lesion exhibits a well-established profile that can be identified even in small samples. In the present study, VEGF was detected in epithelial tissue, as well as in different cells of connective tissue, confirming 
that the expression of this pro-angiogenic agent occurs in different cell types such as keratinocytes, fibroblasts, activated macrophages and inflammatory cells [3]. The literature reports that ANG-1 is expressed in pericytes, smooth muscle cells, fibroblasts and tumor cells and ANG-2 almost exclusively in endothelial and perivascular cells [6]. However, ours results demonstrated that these growth factors are expressed in epithelium and that ANG-2 is also present in connective tissue cells. Chien et al. [22] demonstrated that in specimens of oral squamous cell carcinomas, these markers were predominantly found in the cytoplasm of tumor cells. In chronic inflammatory diseases such as rheumatoid arthritis, the majority of studies were performed by evaluating the serum levels of ANG-1 and ANG-2 [5], thus making it difficult to compare with the type of staining found in our study.

Considering the immunodetection of VEGF, our results revealed that the ROLP group showed significantly higher values in comparison to the AEOLP group. This result is in contrast with that of earlier studies. Tao et al. [13] did not find significant differences in the immunodetection of VEGF between reticular and erosive OLP. Mardani et al. [14], in turn, reported significantly higher serum VEGF levels in patients with atrophic erosive versus reticular OLP. Since VEGF is the principal inducer of angiogenesis and considering that atrophic-erosive lesions represent acute processes of the disease, we expected to see higher expression of this growth factor in the AEOLP group. However recent study has shown that the mean density of the inflammatory infiltrate is higher in reticular lesions than erosive OLP [23], which could explain the increase in VEGF expression in ROLP, since it is known that the expression of this pro-angiogenic growth factor occurs in different cell types, including inflammatory cells [3]. On the other hand, the significantly lower values in the atrophic-erosive lesions can be associated with the use of topical corticosteroids for the pain caused by the lesions.

The utilization of these drugs modulates the inflammatory response, interfering with the expression of VEGF [24]. Still regarding the immunodetection of VEGF, it should be noted that the value found in the OLP group was significantly lower than in the OSCC group, significantly higher than in the OFH group and not differing in relation to the OED group, suggesting that angiogenesis in OLP can behave in a similar way as more aggressive lesions. In study of the Al-Hassiny et al. [25] the expression of pro-angiogenic factors was higher in OLP compared with non-specific oral mucosal inflammatory tissues, indicating that angiogenic factors are differentially expressed in OLP compared with inflamed tissues. There are no reports in the literature about immunodetection of ANG-1 and ANG-2 in lesions of OLP. In this study, the values of these markers did not differ between ROLP and AEOLP groups. The OLP group showed a significantly higher percentage of immunodetection for $\mathrm{ANG}-1$ in relation to the $\mathrm{OFH}$ group. The immunodetection of ANG-2 did not significantly differ between the OLP group and the controls, but very similar values were found in the OLP and OED groups. Comparative studies evaluating the expression of proteins such as p53 and bcl-2 [26] and cell proliferative activity, by investigating PCNA [27], also reported similar values between lesions of OLP and epithelial dysplasia's.
This study has demonstrated a lack of correlation between Ang1, ANG-2 and VEGF in the different groups studied. This finding may be result of the small sample size, but also may reflect the complexity of angiogenesis itself, which can be influenced by a series of other pro-angiogenic cytokines and cell types to determine vascular neoformation. The comparison of the number of vessels did not demonstrate a significant difference between the ROLP and AEOLP groups. Previous studies demonstrated significantly higher values MVD of erosive OLP lesions compared with the lesions of reticular OLP [13,15]. Tao et al. [13] suggested that angiogenesis is correlated with different clinical forms of this disease, but this notion was not supported by our study. In the present research, we chose not to compare the number of vessels between OLP group and control groups, because they involve lesions of different nature, among which some show a histological field with a predominance of epithelial cells while others a predominance of connective tissue, which could have interfered with the reliability of this analysis.

\section{Conclusion}

In this small sample, the results of immunodetection of VEGF, ANG-1 and ANG-2 between ROLP and AEOLP groups indicate that angiogenesis in OLP cannot be associated to the different clinical forms of this disease. The lack of correlation between the markers in the different groups indicates the involvement of other pro-angiogenic agents in the neovascularization and reflects the complexity of this process. Furthermore, immunodetection of growth factors evaluated was similar between OLP and DEO, demonstrating that angiogenesis in OLP may behave similarly to oral epithelial dysplasia and that this disease has different biological behavior of other chronic inflammatory processes benign as fibroepithelial hyperplasia. Further studies are needed to investigate other pro-angiogenic cytokines to better understand the process of angiogenesis in this disease.

\section{Acknowledgment}

This study was financed in part by the Coordenação de Aperfeiçoamento de Pessoal de Nivel Superior-Brasil (CAPES)Finance Code 001.

\section{References}

1. Pandya NM, Dhalla NS, Santini DD (2006) Angiogenesis-a new target for future therapy. Vascular Pharmacol 44(5): 265-274.

2. Weis SM, Cheresh DA (2005) Pathophysiological consequences of VEGFinduced vascular permeability. Nature 437(7058): 497-504.

3. Ferrara N, Gerber HP, Lecouter J (2003) The biology of VEGF and its receptors. Nat Med 9(6): 669-676.

4. Thomas M, Augustin HG (2009) The role of the angiopoietins in vascular morphogenesis. Angiogenesis 12(2): 125-137.

5. Clavel G, Bessis N, Lemeiter D, Fardellone P, Mejjad O, et al. (2007) Angiogenesis markers (VEGF, soluble receptor of VEGF and angiopoietin-1) in very early arthritis and their association with inflammation and joint destruction. Clin Immunol 124(2): 158-164.

6. Fiedler U, Augustin HG (2006) Angiopoietins: a link between angiogenesis and inflammation. Trends Immunol 27(12): 552-558.

7. Fagiani E, Christofori G (2013) Angiopoietins in angiogenesis. Cancer Lett 328(1): 18-26. 
8. Hanahan D (1997) Signaling vascular morphogenesis and maintenance. Science 277(5322): 48-50.

9. Tipoe GL, Jin Y, White FH (1996) The relationship between vascularity and cell proliferation in human normal and pathological lesions of the oral cheek epithelium. Eur J Cancer B Oral Oncol 32B(1): 24-31.

10. Gandolfo M, Keszler A, Lanfranchi H, Itoiz ME (2011) Increased subepithelial vascularization and VEGF expression reveal potentially malignant changes in human oral mucosa lesions. Oral Surg Oral Med Oral Pathol Oral Radiol Endod 111(4): 486-493.

11. Xue JL, Fan MW, Wang SZ, Chen XM, Li Y, et al. (2005) A clinical study of 674 patients with oral lichen planus in China. J Oral Pathol Med 34(8): 467-472.

12. Warnakulasuriya S, Johnson NW, Van der Wall I (2007) Nomenclature and classification of potentially malignant disorders of the oral mucosa. J Oral Pathol Med 36(10): 575-580.

13. Tao X, Huang Y, Li R, Qing R, Ma L, Rhodus Nl et al. (2007) Assessment of local angiogenesis and vascular endotelial growth factor in the patients with atrophic-erosive and reticular oral lichen planus. Oral Surg Oral Med Oral Pathol Oral Radiol Endod 103(5): 661-669.

14. Mardani M, Ghabanchi J, Fattahi MJ, Tadbir AA (2012) Serum level of vascular endothelial growth factor in patients with different clinical subtypes of oral lichen planus. Iran J Med Sci 37(4): 233-237.

15. Hazzaa HHA, El Wakeel NM, Attia EAS, Hager EAA (2016) ALKI expression in oral lichen planus: a possible relation to microvessel density. J Oral Pathol Med 45(5): 373-380.

16. Mittal N, Shankari GM, Palaskar S (2012) Role of angiogenesis in the pathogenesis of oral lichen planus. J Oral Maxillofac Pathol 16(1): 45-48.

17. Scardina GA, Picone V, Cacioppo A, Messina P (2007) Study of microcirculation in oral lichen planus by video-capillaroscopy. Oral Surg Oral Med Oral Pathol Oral Radiol Endod 103(4): e30-4.

18. Pindborg, JJ, Reichart PA, Smith CJ, Van der Wall I (1997) World Health Organization International. Histological Classification of Tumours.
Histological Typing of Cancer and Precancer of the Oral Mucosa. $2^{\text {nd }}$ edn), Springer, Berlin, Germany.

19. Lee JJ, Kuo MY, Cheng SJ, Chiang CP, Jeng JH, et al. (2005) Higher expressions of p53 and proliferating cell nuclear antigen (PCNA) in atrophic oral lichen planus and patients with areca quid chewing. Oral Surg Oral Med Oral Pathol Oral Radiol Endod 99(4): 471-478.

20. Silverman S (2000) Oral lichen planus: a potentially premalignant lesion. J Oral Maxillofac Surg 58(11): 1286-1288.

21. Shen ZY, Liu W, Zhu LK, Feng JQ, Tang GY, et al. (2012) A retrospective clinicopathological study on oral lichen planus and malignant transformation: analysis of 518 cases. Med Oral Patol Oral Cir Bucal 17(6): e943-e947.

22. Chien CY, Su CY, Chuang HC, Fang FM, Huang HY, et al. (2008) Angiopoietin-1 e-2 expression in recurretn squamous cell carcinoma of the oral cavity. J Surg Oncol 97: 273-277.

23. Pereira JS, Monteiro BV, Nonaka CF, Silveira ÉJ, Miguel MC (2012) FoxP3(+) T regulatory cells in oral lichen planus and its correlation with the distinct clinical appearance of the lesions. Int J Exp Pathol 93(4): 287-294.

24. Taylor PC (2005) Serum vascular markers and vascular imaging in assessment of rheumatoid arthritis disease activity and response to therapy. Rheumatology (Oxford) 44(6): 721-728.

25. Al Hassiny A, Friedlander LT, Parachuru VPB, Seo B, Hussaini HM, et al. (2018) Upregulation of angiogenesis in oral lichen planus. J Oral Pathol Med 47(2): 173-178.

26. Sousa FA, Paradella TC, Carvalho YR, Rosa LE (2009) Immunohistochemical expression of PCNA, p53, bax and bcl-2 in oral lichen planus and epithelial dysplasia. J Oral Sci 51(1): 117-121.

27. de Sousa FA, Paradella TC, Carvalho YR, Rosa LE (2009) Comparative analysis of cell proliferation ratio in oral lichen planus, epithelial dysplasia and oral squamous cell carcinoma. Med Oral Patol Oral Circ Bucal 14(11): e563-e567. 